\title{
Impact of right-handed interactions on the propagation of Dirac and Majorana neutrinos in matter
}

\author{
F. del Aguila,* \\ Departamento de Física Teórica y del Cosmos and \\ Centro Andaluz de Física de Partículas Elementales (CAFPE), \\ Universidad de Granada, E-18071 Granada, Spain \\ J. Syskdt and M. Zrałekt \\ Department of Field Theory and Particle Physics, Institute of Physics, \\ University of Silesia, Uniwersytecka 4, 40-007 Katowice, Poland
}

\begin{abstract}
Dirac and Majorana neutrinos can be distinguished in relativistic neutrino oscillations if new right-handed interactions exist, due to their different propagation in matter. We review how these new interactions affect neutrino oscillation experiments and discuss the size of this eventually observable effect for different oscillation channels, baselines and neutrino energies.
\end{abstract}

*Electronic address: faguila@ugr.es

${ }^{\dagger}$ Electronic address: jacek@server.phys.us.edu.pl

${ }^{\ddagger}$ Electronic address: zralek@us.edu.pl 


\section{INTRODUCTION}

One of the fundamental mysteries of neutrino physics is the nature of massive neutrinos 1]. They can have Dirac or Majorana masses [2], with their own signatures in each case. In particular, it is assumed that the best way to establish their character is to search for neutrinoless double $\beta$ decay $\left((\beta \beta)_{0 \nu}\right)[\underline{3}, 4,5]$. Indeed, its mere observation would imply that neutrinos are Majorana particles. However, if neutrino masses are a fraction of eV and no New Physics (NP) gives large enough contributions, it will be quite difficult to observe $(\beta \beta)_{0 \nu}[\underline{6}, \underline{7}, \underline{8}, \underline{9}]$.

Although oscillation experiments are not sensitive to the absolute scale of neutrino masses, they are able to probe very tiny neutrino mass differences [10, 11, 12]. Hence, the obvious question is if neutrino oscillations can distinguish between Dirac and Majorana neutrino

masses [13, 14]. This is not possible in neutrino oscillations in vacuum [15, 16]. The reason is that the additional phases in the neutrino mixing matrix distinguishing Majorana from Dirac neutrinos disappear from the oscillation probability expressions. The oscillation in matter can differ from that in vacuum, leading to both the change of the effective mixing angles and of the effective masses [17, 18, 19]. However, if neutrinos are relativistic and only interact through left-handed $(\mathrm{LH})$ currents, the oscillation in matter does not discriminate between both types of fermions either. This is not the case for non-relativistic neutrinos [20] but unfortunately these neutrinos are not available in any experiment, at least up to now. So, within the New Standard Model $(\nu S M)[21]$ and for neutrinos in the mass range of tens of meV it will be very difficult to find experimental evidence which can distinguish Dirac from Majorana neutrinos.

Then, one may wonder if there is some type of NP which can modify neutrino propagation in a dense medium [22, 23, 24, 25, 26] to allow for the determination of the neutrino mass character. As we will review, although NP can give rise to a low energy Hamiltonian with any tensor structure for neutrino bilinears $\bar{\nu} \Gamma^{a} \nu$, with $\Gamma^{a}=I, \gamma_{5}, \gamma^{\mu}, \gamma^{\mu} \gamma_{5}$, and $\sigma^{\mu \nu}$, the scalar and pseudoscalar currents vanish or are suppressed for relativistic neutrinos. They are $0\left(\frac{m}{E}\right)$, where $m$ is a light neutrino mass and $E$ the neutrino energy. Vector and axialvector helicity flip and tensor helicity non-flip transitions have similar suppression factors. Only the vector and axial-vector helicity non-flip transitions and the tensor helicity flip ones are unsuppressed, and may then help to discriminate between relativistic Dirac and 
Majorana neutrinos propagating in a dense medium. Here we will concentrate on the spin non-flip vector and axial-vector transitions. Tensor interactions have been discussed in this context in [27]. In any case, as the production and detection of neutrinos (antineutrinos) with positive (negative) helicity are strongly suppressed, the sensitivity to spin-flip tensor transitions is reduced. Besides, they require a polarised background to manifest, and if generated at higher orders in perturbation theory, they are also extra suppressed by loop factors $^{1}$ [28].

In this paper we will consider an effective Lagrangian with only arbitrary vector and axial-vector couplings modifying the LH structure of the $\nu S M$. This is enough to generate (new) vector and axial-vector four-fermion interactions which are those to be probed by relativistic neutrinos. The new couplings affect differently the propagation of Dirac and Majorana neutrinos in matter. The largest effect results from the addition of new righthanded $(\mathrm{RH})$ neutral currents, being the corresponding transition probability dependence linear ${ }^{2}$. We discuss its size for different channels as a function of the neutrino energy and the baseline, showing that it can eventually allow for discriminating between Dirac and Majorana neutrinos.

We first revise the necessary known results on neutrino propagation in a dense medium. In next Section we describe the neutrino interaction with matter introducing a general, relativistic invariant Hamiltonian, and show that for relativistic neutrinos only vector, axialvector and tensor terms have contributions to neutrino oscillation amplitudes which are not suppressed by $\frac{m}{E}$ factors. In Section III we discuss the Lagrangian with general LH and RH currents. As deviations from the $\nu S M$ are strongly constrained by existing experimental data, they must be small. Then, using the corresponding four-fermion effective Hamiltonian we calculate the effective interaction with matter for Dirac and Majorana neutrinos. In Section [V] the probabilities for neutrino oscillations in matter are derived for a neutral background with uniform spin and momentum distributions [29]. We present our numerical results for neutrino propagation inside Earth for different channels, baselines and neutrino

\footnotetext{
${ }^{1}$ They also result from Fierz rearrangement of (pseudo)scalar interactions and can be of the same order as the other effective four-fermion interactions, being only suppressed by the corresponding inverse power of the large effective mass scale and eventually by the product of small (Yukawa) couplings.

${ }^{2}$ Although leading order terms in the effective Lagrangian expansion may be actually subdominant in definite models.
} 
energies in Section V. Finally, last Section is devoted to conclusions and to comment on some necessary ingredients of more fundamental models with RH neutrino interactions.

\section{DIRAC VERSUS MAJORANA NEUTRINOS}

Let us evaluate the suppression factors for the different transitions. The effective Hamiltonian describing the coherent neutrino scattering in a dense medium has the form

$$
\mathcal{H}^{i n t}=\sum_{a} \sum_{i, j}\left[\left(z_{a}\right)_{i j}\left(\bar{\nu}_{i} \Gamma^{a} \nu_{j}\right)+\left(z_{a}^{*}\right)_{i j}\left(\bar{\nu}_{j} \bar{\Gamma}^{a} \nu_{i}\right)\right]
$$

where $\Gamma^{a}=I, \gamma_{5}, \gamma^{\mu}, \gamma^{\mu} \gamma_{5}$ and $\sigma^{\mu \nu}=\frac{i}{2}\left[\gamma^{\mu}, \gamma^{\nu}\right]$, and $z_{a}$ are the complex scalar $\left(z_{S}\right)$, pseudoscalar $\left(z_{P}\right)$, vector $\left(z_{V \mu}\right)$, axial-vector $\left(z_{A \mu}\right)$ and tensor $\left(z_{T \mu \nu}\right)$ matrices characterizing the background. The sum $i, j$ runs over mass eigenstates. For the moment it is enough to know that the Hamiltonian is hermitian, what follows from the relations

$$
\bar{\Gamma}^{a} \equiv \gamma_{0}\left(\Gamma^{a}\right)^{\dagger} \gamma_{0}=\left\{\begin{aligned}
\Gamma^{a}, & \text { for } a=S, V, A, T \\
-\Gamma^{a}, & \text { for } a=P .
\end{aligned}\right.
$$

The $j \rightarrow i$ matrix element

$$
\mathcal{H}_{i j}=\left\langle\nu_{i}\left|\mathcal{H}^{i n t}\right| \nu_{j}\right\rangle
$$

can be calculated using the plane wave decomposition for the neutrino fields and contracting the corresponding creation and annihilation operators with those of the initial and final Dirac neutrino, Dirac antineutrino or Majorana neutrino states. We obtain for each case

$$
\begin{aligned}
\mathcal{H}_{i j}^{D} & =\sum_{a}\left[\left(z_{a}\right)_{i j} \bar{u}_{i} \Gamma^{a} u_{j}+\left(z_{a}^{*}\right)_{j i} \bar{u}_{i} \bar{\Gamma}^{a} u_{j}\right], \\
\mathcal{H}_{i j}^{D} & =-\sum_{a}\left[\left(z_{a}\right)_{j i} \bar{v}_{j} \Gamma^{a} v_{i}+\left(z_{a}^{*}\right)_{i j} \bar{v}_{j} \bar{\Gamma}^{a} v_{i}\right], \\
\mathcal{H}_{i j}^{M} & =\mathcal{H}_{i j}^{D}+\mathcal{H} \frac{D}{i j},
\end{aligned}
$$

respectively. Yet it is more convenient to rewrite them only as a function of positive frequency spinors using the charge conjugation relation $v_{i}=C \bar{u}_{i}^{T}$, together with Eq. (2) and the relations

$$
C\left(\Gamma^{a}\right)^{T} C^{-1}=\left\{\begin{aligned}
\Gamma^{a}, & \text { for } a=S, P, A, \\
-\Gamma^{a}, & \text { for } a=V, T .
\end{aligned}\right.
$$


Then, they read

$$
\begin{aligned}
\mathcal{H}_{i j}^{D}= & \left(Z_{S}\right)_{i j} \bar{u}_{i} u_{j}+\left(Z_{P}\right)_{i j} \bar{u}_{i} \gamma_{5} u_{j}+\left(Z_{V \mu}\right)_{i j} \bar{u}_{i} \gamma^{\mu} u_{j} \\
& +\left(Z_{A \mu}\right)_{i j} \bar{u}_{i} \gamma^{\mu} \gamma_{5} u_{j}+\left(Z_{T \mu \nu}\right)_{i j} \bar{u}_{i} \sigma^{\mu \nu} u_{j}, \\
\mathcal{H}_{i j}^{D}= & \left(Z_{S}^{*}\right)_{i j} \bar{u}_{i} u_{j}-\left(Z_{P}^{*}\right)_{i j} \bar{u}_{i} \gamma_{5} u_{j}-\left(Z_{V \mu}^{*}\right)_{i j} \bar{u}_{i} \gamma^{\mu} u_{j} \\
& +\left(Z_{A \mu}^{*}\right)_{i j} \bar{u}_{i} \gamma^{\mu} \gamma_{5} u_{j}-\left(Z_{T \mu \nu}^{*}\right)_{i j} \bar{u}_{i} \sigma^{\mu \nu} u_{j}, \\
\mathcal{H}_{i j}^{M}= & 2\left(\operatorname{Re} Z_{S}\right)_{i j} \bar{u}_{i} u_{j}+2 i\left(\operatorname{Im} Z_{P}\right)_{i j} \bar{u}_{i} \gamma_{5} u_{j}+2 i\left(\operatorname{Im} Z_{V \mu}\right)_{i j} \bar{u}_{i} \gamma^{\mu} u_{j} \\
& +2\left(\operatorname{Re} Z_{A \mu}\right)_{i j} \bar{u}_{i} \gamma^{\mu} \gamma_{5} u_{j}+2 i\left(\operatorname{Im} Z_{T \mu \nu}\right)_{i j} \bar{u}_{i} \sigma^{\mu \nu} u_{j},
\end{aligned}
$$

where the capital $Z$ matrices are constructed from the small $z$ ones. They are hermitian $Z=z+z^{\dagger}$ for $\mathrm{S}, \mathrm{V}, \mathrm{A}, \mathrm{T}$ and antihermitian $Z=z-z^{\dagger}$ for $\mathrm{P}$ terms. As we shall see, Eq. (5) is further simplified for relativistic neutrinos.

\section{A. Relativistic limit}

In the Weyl representation the spinor $u_{\lambda}\left(p_{\nu}\right)$ for a particle with helicity $\frac{\lambda}{2}$, energy $E_{\nu}$ and momentum $p_{\nu}$, and normalized in such a way that $u^{\dagger} u=1$, has the form

$$
u_{\lambda}\left(p_{\nu}\right)=\left(\begin{array}{l}
\sqrt{\frac{E_{\nu}+\lambda\left|\vec{p}_{\nu}\right|}{2 E_{\nu}}} \chi_{\lambda} \\
\sqrt{\frac{E_{\nu}-\lambda\left|\vec{p}_{\nu}\right|}{2 E_{\nu}}} \chi_{\lambda}
\end{array}\right),
$$

where $\chi_{\lambda}$ is the Pauli helicity spinor properly normalized $\chi^{\dagger} \chi=1$. In order to compare the spinor products for relativistic neutrinos with different masses, it is convenient to use a common energy, which is independent of the particular small neutrino masses involved.

Indeed, noting that in forward neutrino scattering all momenta are parallel $\vec{p}_{i}=p_{i} \vec{i}$ and following [30], we can write the energy and the momentum of any light neutrino

$$
E_{i}=E+\xi \frac{m_{i}^{2}}{2 E}, \quad p_{i}=E-(1-\xi) \frac{m_{i}^{2}}{2 E},
$$

where $\xi$ is some parameter which depends on the production or detection process, and $E$ is the neutrino energy for zero neutrino mass. Using these expressions we can evaluate the 
spinor products entering in Eq. (15)

$$
\begin{array}{ll}
\bar{u}_{i}(\lambda) u_{j}(\lambda) & =\frac{m_{i}+m_{j}}{2 E}+0\left(\left(\frac{m}{E}\right)^{3}\right) \\
\bar{u}_{i}(\lambda) u_{j}(-\lambda) & =0 \\
\bar{u}_{i}(\lambda) \gamma^{5} u_{j}(\lambda) & =\lambda \frac{m_{i}-m_{j}}{2 E}+0\left(\left(\frac{m}{E}\right)^{3}\right) \\
\bar{u}_{i}(\lambda) \gamma^{5} u_{j}(-\lambda) & =0 \\
\bar{u}_{i}(\lambda) \gamma^{\mu} u_{j}(\lambda) & =n^{\mu}+0\left(\left(\frac{m}{E}\right)^{2}\right) \\
\bar{u}_{i}(-1) \gamma^{\mu} u_{j}(+1) & =-\left[\bar{u}_{i}(+1) \gamma^{\mu} u_{j}(-1)\right]^{*}=m^{\mu} \frac{m_{i}-m_{j}}{2 E}+0\left(\left(\frac{m}{E}\right)^{3}\right), \\
\bar{u}_{i}(\lambda) \gamma^{\mu} \gamma_{5} u_{j}(\lambda) & =\lambda n^{\mu}+0\left(\left(\frac{m}{E}\right)^{2}\right) \\
\bar{u}_{i}(-1) \gamma^{\mu} \gamma_{5} u_{j}(+1) & =\left[\bar{u}_{i}(+1) \gamma^{\mu} \gamma_{5} u_{j}(-1)\right]^{*}=m^{\mu} \frac{m_{i}+m_{j}}{2 E}+0\left(\left(\frac{m}{E}\right)^{3}\right), \\
\bar{u}_{i}(\lambda) \sigma^{0 k} u_{j}(\lambda) & =i n^{k} \frac{m_{i}-m_{j}}{2 E}+0\left(\left(\frac{m}{E}\right)^{3}\right), \\
\bar{u}_{i}(\lambda) \sigma^{k l} u_{j}(\lambda) & =\lambda \varepsilon^{k l r} n^{r} \frac{m_{i}+m_{j}}{2 E}+0\left(\left(\frac{m}{E}\right)^{3}\right) \\
\bar{u}_{i}(-1) \sigma^{0 k} u_{j}(+1) & =\left[\bar{u}_{i}(+1) \sigma^{0 k} u_{j}(-1)\right]^{*}=i m^{k}+0\left(\left(\frac{m}{E}\right)^{2}\right) \\
\bar{u}_{i}(-1) \sigma^{k l} u_{j}(+1) & =\left[\bar{u}_{i}(+1) \sigma^{k l} u_{j}(-1)\right]^{*}=\varepsilon^{k l r} m^{r}+0\left(\left(\frac{m}{E}\right)^{2}\right),
\end{array}
$$

where $n^{\mu}=(1, \vec{n})$ with $\vec{n}=(\sin \theta \cos \varphi, \sin \theta \sin \varphi, \cos \theta)$ the direction of the neutrino momentum, and $m^{\mu}=(0, \vec{m})$ with $\vec{m}=(\cos \theta \cos \varphi-i \sin \varphi, \cos \theta \sin \varphi+i \cos \varphi,-\sin \theta)$ orthogonal to $\vec{n}$ and defined up to an unphysical global phase; whereas $\varepsilon^{k l r}$ is the totally antisymmetric tensor with $\varepsilon^{123}=1$.

Hence, we see that in the relativistic limit for forward neutrino scattering the scalar and pseudoscalar terms can be effectively omitted. If in addition we assume that the tensor term is negligible (the general case including non-vanishing tensor interactions will be considered elsewhere), then not only the corresponding suppressed spin non-flip but the spin flip terms can be omitted, and Eq. (5) reduces to

$$
\begin{aligned}
\mathcal{H}_{i j}^{D}(\lambda) & =\left(Z_{V \mu}+\lambda Z_{A \mu}\right)_{i j} n^{\mu}, \\
\mathcal{H} & \frac{D}{i j}(\lambda)=\left(-Z_{V \mu}^{*}+\lambda Z_{A \mu}^{*}\right)_{i j} n^{\mu}, \\
\mathcal{H}_{i j}^{M}(\lambda) & =2\left(i \operatorname{Im} Z_{V \mu}+\lambda \operatorname{Re} Z_{A \mu}\right)_{i j} n^{\mu} .
\end{aligned}
$$

Thus, the allowed transitions do not flip helicity and depend on the vector and axial-vector couplings only. In order to answer the question on the nature of the neutrino mass we must 
compare the amplitudes $\mathcal{H}_{i j}^{D}(\lambda=-1)$ with $\mathcal{H}_{i j}^{M}(\lambda=-1)$ for particles, and $\mathcal{H} \frac{D}{i j}(\lambda=+1)$ with $\mathcal{H}_{i j}^{M}(\lambda=+1)$ for antiparticles.

In the $\nu S M$, where neutrinos interact only through LH currents, the vector and axialvector $Z$ matrices are related, $Z_{V \mu}=-Z_{A \mu}$, and the only non-vanishing transitions read

$$
\begin{aligned}
& \mathcal{H}_{i j}^{D}(\lambda=-1)=\mathcal{H}_{i j}^{M}(\lambda=-1)=2\left(Z_{V \mu}\right)_{i j} n^{\mu}, \\
& \mathcal{H}_{i j}^{D}(\lambda=+1)=\mathcal{H}_{i j}^{M}(\lambda=+1)=-2\left(Z_{V \mu}^{*}\right)_{i j} n^{\mu} .
\end{aligned}
$$

In any model where neutrinos besides interact through $\mathrm{RH}$ currents $Z_{V \mu} \neq-Z_{A \mu}$, and there are deviations from the $\nu S M$ predictions in Eq. (10) which also affect differently the Dirac and Majorana neutrino propagation. Indeed, Eq. (9) gives our main input

$$
\begin{aligned}
& \mathcal{H}_{i j}^{D}(\lambda=-1)-\mathcal{H}_{i j}^{M}(\lambda=-1)=\left(Z_{V \mu}^{*}+Z_{A \mu}^{*}\right)_{i j} n^{\mu}, \\
& \mathcal{H} \frac{D}{i j}(\lambda=+1)-\mathcal{H}_{i j}^{M}(\lambda=+1)=-\left(Z_{V \mu}+Z_{A \mu}\right)_{i j} n^{\mu} .
\end{aligned}
$$

Hence, even in the relativistic limit there is in principle the possibility of distinguishing between the Dirac and Majorana neutrinos, but only if besides the dominant LH current there is some RH piece. Whether this can have practical consequences, it depends on the strength of the RH interaction.

\section{LAGRANGIAN AND EFFECTIVE LOW-ENERGY HAMILTONIAN DENSI- TIES}

One may adopt a more phenomenological approach at this point and parameterise the difference between Dirac and Majorana amplitudes in Eq. (11), calculating the corresponding oscillation probabilities afterwards and looking for the largest possible effects. However, one must also worry about the physical implications of such a parameterisation and the experimental constraints that restrict the different parameters. Then, it is more enlightening to start extending the $\nu S M$ Lagrangian describing the coherent scattering of neutrinos on background fermions $f$,

$$
\nu_{j}+f \rightarrow \nu_{i}+f .
$$

Before discussing any specific model, let us introduce an effective Lagrangian with arbitrary $\mathrm{LH}$ and $\mathrm{RH}$ currents and show that the largest effects are associated to new RH neutral currents involving the light neutrinos. 


\section{A. The effective left-right interaction Lagrangian}

The $\nu S M$ charged current Lagrangian can be generalised to include new LH and RH couplings

$$
\mathcal{L}_{C C}=-\frac{e}{2 \sqrt{2} \sin \theta_{W}} \sum_{i, \alpha} \bar{\nu}_{i} \gamma^{\mu}\left[\varepsilon_{L}^{C}\left(1-\gamma_{5}\right)+\delta_{R}^{C}\left(1+\gamma_{5}\right)\right] U_{\alpha i}^{*} l_{\alpha} W_{\mu}^{+}+\text {h.c. }
$$

where $\varepsilon_{L}^{C}$ and $\delta_{R}^{C}$ are taken to be global factors deviating slightly from their $\nu S M$ values, 1 and 0, respectively. This is enough for as we will show, they enter quadratically in the effective interaction Hamiltonian. So, $\left|\delta_{R}^{C}\right|^{2}$ contributions are negligible because we are interested in large (linear) effects. Then, although in contrast with Eq. (12) the RH and LH charged currents can have in general different mixing matrices, its explicit form will not matter in the RH case. While $\left|\varepsilon_{L}^{C}\right|^{2}$ terms contribute the same to the propagation of Dirac and Majorana neutrinos. Thus, we will take $\varepsilon_{L}^{C}=1$ and $U_{\alpha i}$ to be the $3 \times 3$ unitary matrix giving the linear combinations of mass eigenstates with well-defined lepton flavour [31, 32]

$$
\nu_{\alpha}=\sum_{i} U_{\alpha i} \nu_{i}
$$

One must keep in mind, however, that in definite models the terms neglected can be of the same order as those distinguishing between Dirac and Majorana neutrinos below.

Similarly, the neutral current Lagrangian can be written

$$
\begin{aligned}
\mathcal{L}_{N C} & =-\frac{e}{4 \sin \theta_{W} \cos \theta_{W}}\left\{\sum_{i, j} \bar{\nu}_{i} \gamma^{\mu}\left[\varepsilon_{L}^{N \nu}\left(1-\gamma_{5}\right) \delta_{i j}+\delta_{R}^{N \nu}\left(1+\gamma_{5}\right) \Omega_{i j}^{R}\right] \nu_{j}+\right. \\
& \left.+\sum_{f} \bar{f} \gamma^{\mu}\left[\varepsilon_{L}^{N f}\left(1-\gamma_{5}\right)+\varepsilon_{R}^{N f}\left(1+\gamma_{5}\right)\right] f\right\} Z_{\mu},
\end{aligned}
$$

where as before $\varepsilon_{L}^{N \nu}$ is a global factor of order 1 , its $\nu S M$ value, to keep track of the order of the different terms. The LH piece for light neutrinos can be assumed to be diagonal (and $\varepsilon_{L}^{N \nu}=1$ ) because it will not help to determine their nature ${ }^{3}$. What is crucial is the general form of the $\mathrm{RH}$ piece, that we parameterised with a global factor $\delta_{R}^{N \nu}$ of order 0 , the value in the $\nu S M$, multiplying an otherwise arbitrary hermitian matrix $\Omega_{i j}^{R}$. The background fermions can be taken to be $f=e, u, d$ or $e, p, n$, with their neutral couplings

$$
\varepsilon_{L}^{N f}=2 T_{f}^{3}-q_{f} \sin ^{2} \theta_{W}+\delta_{L}^{f}, \quad \varepsilon_{R}^{N f}=-q_{f} \sin ^{2} \theta_{W}+\delta_{R}^{f}
$$

\footnotetext{
${ }^{3}$ In models with neutrino mixing with $\nu S M$ singlets there are (off-diagonal) terms proportional to this mixing and coupling mass eigenstates mainly participating of standard neutrinos and of $\nu S M$ singlets, respectively; as well as quadratic terms correcting the standard couplings.
} 
where $q_{f}\left(T_{f}^{3}\right)$ is the fermion charge (third component of the weak isospin) and $\delta_{L, R}^{f}$ are possible small deviations from their standard values.

The new RH coupling in Eq. (13) requires the addition of $\mathrm{RH}$ neutrinos transforming non-trivially under the $\nu S M$ gauge group, or the $Z$ mixing with an extra gauge boson coupling to the new RH neutrinos if these are $\nu S M$ singlets. Both scenarios have further consequences. For example, RH neutrinos in non-trivial $S U(2)_{L}$ representations imply new charged leptons which have not been observed, and the $Z$ mixing with an extra gauge boson is strongly constrained by processes not involving neutrinos. Definite models must evade these constraints. This is not a problem in the Dirac case, but the observed neutrinos must mix with the new light neutrinos entering in the RH neutral current if the Majorana neutrinos have to feel a different interaction when travelling through matter. Indeed, if there are only three light neutrinos feeling the $\nu S M$ interactions, their nature can not be established in oscillation experiments with very energetic neutrinos, although there are new sectors beyond the $\nu S M$. In order to manifest the Majorana character the light neutrinos must participate of new interactions and then of new degrees of freedom ${ }^{4}$. In either case light neutrinos can mixed with new heavy neutrinos [33, 34], but this mixing which must be rather small is of no relevance to decide about the nature of light neutrinos in oscillation experiments. What matters is the mixing with new light neutrinos with RH interactions. The experimental limits on such a mixing are not so stringent, because all light neutrinos are produced in the standard decay processes and there is no deficit relative to the corresponding $\nu S M$ prediction. On the other hand, in loop processes we have also to sum over all light degrees of freedom, and the new contributions are proportional to the (new) light neutrino mass differences.

We could also think in adding new (pseudo)scalar interactions to generate an effective low energy Hamiltonian with RH neutrino currents coupled to background fermions, but they are also strongly constrained if, as we need, the new interaction couples the observed neutrinos to matter fermions. Besides, some (neutrino) mixing with new fermionic degrees of freedom and between different spin 0 bosons are still necessary.

\footnotetext{
${ }^{4}$ Dirac neutrinos require $\mathrm{RH}$ counterparts, but it is not required that these have other interactions.
} 


\section{B. The general effective interaction Hamiltonian}

The effective low energy four-fermion Hamiltonian resulting from the former charged and neutral interaction Lagrangians has the general form

$$
\mathcal{H}_{e f f}=\sum_{f=e, p, n} \frac{G_{F}}{\sqrt{2}} \sum_{i, j} \sum_{a=V, A}\left(\bar{\nu}_{i} \Gamma^{a} \nu_{j}\right)\left[\bar{f} \Gamma_{a}\left(g_{f a}^{i j}+\bar{g}_{f a}^{i j} \gamma_{5}\right) f\right],
$$

where we neglect the scalar and pseudoscalar terms generated by Fierz rearrangement because for relativistic neutrinos they vanish or are suppressed by small $\frac{m}{E}$ factors, as discussed in Section II. Thus, we are only left with vector and axial-vector interactions, $\Gamma^{a}=\gamma^{\mu}, \gamma^{\mu} \gamma_{5}$, with couplings $g_{f a}^{i j}$ and $\bar{g}_{f a}^{i j}$ given by

$$
\begin{array}{ll}
g_{f V}^{i j}=g_{f L}^{i j}+g_{f R}^{i j}, & \bar{g}_{f V}^{i j}=\bar{g}_{f L}^{i j}+\bar{g}_{f R}^{i j}, \\
g_{f A}^{i j}=-\bar{g}_{f L}^{i j}+\bar{g}_{f R}^{i j}, & \bar{g}_{f A}^{i j}=-g_{f L}^{i j}+g_{f R}^{i j},
\end{array}
$$

where we introduce $\mathrm{LH}$ and $\mathrm{RH}$ couplings for later convenience, and

$$
\begin{array}{ll}
g_{f L}^{i j}=\left(A^{L L}+A^{L R}\right)_{i j}^{f}, & \bar{g}_{f L}^{i j}=\left(-A^{L L}+A^{L R}\right)_{i j}^{f}, \\
g_{f R}^{i j}=\left(A^{R R}+A^{R L}\right)_{i j}^{f}, & \bar{g}_{f R}^{i j}=\left(A^{R R}-A^{R L}\right)_{i j}^{f},
\end{array}
$$

with

$$
\begin{aligned}
& \left(A^{L L}\right)_{i j}^{f}=\left|\varepsilon_{L}^{C}\right|^{2} U_{e i}^{*} U_{e j} \delta_{f e}+\frac{\varrho}{2} \varepsilon_{L}^{N \nu} \varepsilon_{L}^{N f} \delta_{i j}, \quad\left(A^{L R}\right)_{i j}^{f}=\frac{\varrho}{2} \varepsilon_{L}^{N \nu} \varepsilon_{R}^{N f} \delta_{i j}, \\
& \left(A^{R R}\right)_{i j}^{f}=\left|\delta_{R}^{C}\right|^{2} U_{e i}^{*} U_{e j} \delta_{f e}+\frac{\varrho}{2} \delta_{R}^{N \nu} \varepsilon_{R}^{N f} \Omega_{i j}^{R},\left(A^{R L}\right)_{i j}^{f}=\frac{\varrho}{2} \delta_{R}^{N \nu} \varepsilon_{L}^{N f} \Omega_{i j}^{R},
\end{aligned}
$$

where $\varrho=\frac{M_{W}^{2}}{M_{Z}^{2} \cos ^{2} \theta_{W}} \simeq 1$. For comparison, within the $\nu S M$ only

$$
g_{f V}^{i j}=-\bar{g}_{f A}^{i j} \quad \text { and } \quad \bar{g}_{f V}^{i j}=-g_{f A}^{i j}
$$

are different from zero, reproducing the well-known results for LH neutrino interactions. The form of the Hamiltonian in Eq. (15) [17, 27] is especially useful for our purposes. As neutrinos and background fermions $f=e, p, n$ are placed in two separated factors, it is straightforward to derive the coherent neutrino scattering in matter summing over all fermions and averaging over their properties [32]. This is what is done when going from Eq. (15) to Eq. (11) [33]

$$
\begin{aligned}
& \left(z_{V}^{\mu}\right)_{i j}+\left(z_{V}^{*}\right)_{j i}=\left(Z_{V}^{\mu}\right)_{i j}=\frac{G_{F}}{\sqrt{2}} \sum_{f} N_{f}\left(g_{f V}^{i j}\left\langle\frac{p_{f}^{\mu}}{E_{f}}\right\rangle+m_{f} \bar{g}_{f V}^{i j}\left\langle\frac{s_{f}^{\mu}}{E_{f}}\right\rangle\right), \\
& \left(z_{A}^{\mu}\right)_{i j}+\left(z_{A}^{*}\right)_{j i}=\left(Z_{A}^{\mu}\right)_{i j}=\frac{G_{F}}{\sqrt{2}} \sum_{f} N_{f}\left(\bar{g}_{f A}^{i j}\left\langle\frac{\left\langle_{f}^{\mu}\right.}{E_{f}}\right\rangle+m_{f} g_{f A}^{i j}\left\langle\frac{s_{f}^{\mu}}{E_{f}}\right\rangle\right),
\end{aligned}
$$


where $N_{f}$ with $f=e, p, n$ stand for the number of fermions $f$ per unit volume, and $\left\langle\frac{p_{f}^{\mu}}{E_{f}}\right\rangle$ and $\left\langle\frac{s_{f}^{\mu}}{E_{f}}\right\rangle$ are the properly normalised averages over the corresponding fermion distributions of the momentum and the spin, respectively.

\section{The effective, coherent neutrino interaction in matter}

The neutrino background coherent scattering is now easy to calculate from the effective four-fermion Hamiltonian. Propagation of the light, relativistic neutrinos in matter is governed by an evolution equation of the Schrödinger type for each spinor helicity for this is conserved (see e.g. [33, 35]):

$$
i \frac{d}{d t} \Psi_{i}^{\nu}(\vec{p}, t)=\sum_{j} \mathcal{H}_{i j}^{\nu} \Psi_{j}^{\nu}(\vec{p}, t),
$$

where $\Psi_{i}^{\nu}(\vec{p}, t)$ is the wave function for the neutrino (antineutrino) eigenstate of mass $m_{i}$, momentum $\vec{p}$ and helicity $\frac{\lambda}{2}=-\frac{1}{2}\left(+\frac{1}{2}\right)$. As usual [30, 32], we assume that all particles have the same momentum but different energies $E_{i}=\sqrt{\vec{p}^{2}+m_{i}^{2}}$. Then, the effective Hamitonian in Eq. (21) reads

$$
\mathcal{H}_{i j}^{\nu}=\left(p+\frac{m_{i}^{2}}{2 p}\right) \delta_{i j}+\mathcal{H}_{i j},
$$

where $\mathcal{H}_{i j}$ describes the coherent neutrino scattering inside matter, thus depending on the properties of the medium which is characterised by the fermion contents and the corresponding polarization, momentum and charge distributions. As it is apparent from Eqs. (9) and (20), $\mathcal{H}_{i j}$ is also different for Dirac and Majorana neutrinos. In the case of a medium unpolarized, isotropic and neutral $\left(N_{e}=N_{p}\right)$, the Hamiltonian for Dirac neutrinos with helicity $\frac{\lambda}{2}=-\frac{1}{2}$ is

$$
\mathcal{H}_{i j}^{D}(\lambda=-1)=\sqrt{2} G_{F}\left[N_{e}\left(g_{e L}^{i j}+g_{p L}^{i j}\right)+N_{n} g_{n L}^{i j}\right]
$$

whereas for Majorana neutrinos of the same helicity it reads

$$
\mathcal{H}_{i j}^{M}(\lambda=-1)=\sqrt{2} G_{F}\left[N_{e}\left(g_{e L}^{i j}-g_{e R}^{* i j}+g_{p L}^{i j}-g_{p R}^{* i j}\right)+N_{n}\left(g_{n L}^{i j}-g_{n R}^{* i j}\right)\right] .
$$

Thus, Dirac and Majorana neutrinos propagate differently in matter in the presence of extra RH interactions, $g_{f R} \neq 0$. Their evolution depends on the matter densities $N_{f}$ and the 
neutrino couplings to electrons, protons and neutrons $g_{f}$. These are related to the $u$ and $d$ quark couplings in the usual way

$$
\begin{array}{ll}
g_{p L}^{i j}=2 g_{u L}^{i j}+g_{d L}^{i j}, & g_{p R}^{i j}=2 g_{u R}^{i j}+g_{d R}^{i j} \\
g_{n L}^{i j}=g_{u L}^{i j}+2 g_{d L}^{i j}, & g_{n R}^{i j}=g_{u R}^{i j}+2 g_{d R}^{i j} .
\end{array}
$$

The appropriate Hamitonians for Dirac antineutrinos and Majorana neutrinos of positive helicity follow from the well-known relations [33]

$$
\mathcal{H} \frac{D}{i j}(\lambda)=-\left[\mathcal{H}_{i j}^{D}(-\lambda)\right]^{*}, \quad \mathcal{H}_{i j}^{M}(\lambda)=-\left[\mathcal{H}_{i j}^{M}(-\lambda)\right]^{*},
$$

which can be also deduced from Eq. (9). We should remember that these relations are valid in the absence of scalar, pseudoscalar and tensor interactions, for relativistic neutrinos, and in the mass eigenstate basis. In the flavour basis these relations are in general not satisfied.

\section{PROPAGATION OF DIRAC AND MAJORANA NEUTRINOS IN MATTER}

In order to estimate the difference between Dirac and Majorana neutrino oscillations we shall consider the simple case discussed in the previous Section of an unpolarized, isotropic and neutral medium with constant fermion densities $N_{f}$. Then, the neutrino evolution equation in Eq. (21) can be solved analytically. After substracting the non-important common diagonal pieces, the Hamiltonians (23) and (24) can be written in the flavour eigenstate basis

$$
\mathcal{H}_{\alpha \beta}^{D}(\lambda=-1)=\sqrt{2} G_{F} N_{e} \delta_{e \alpha} \delta_{e \beta}
$$

and

$$
\begin{aligned}
\mathcal{H}_{\alpha \beta}^{M}(\lambda=-1)= & \sqrt{2} G_{F}\left\{N_{e} \delta_{e \alpha} \delta_{e \beta}-N_{e}\left[\left|\delta_{R}^{C}\right|^{2} \delta_{e \alpha} \delta_{e \beta}\right.\right. \\
& \left.+\frac{1}{2} \delta_{R}^{N \nu *}\left(\delta_{L}^{e *}+\delta_{R}^{e *}+2 \delta_{L}^{u *}+2 \delta_{R}^{u *}+\delta_{L}^{d *}+\delta_{R}^{d *}\right) \Omega_{\alpha \beta}^{R *}\right] \\
& \left.-N_{n} \frac{1}{2} \delta_{R}^{N \nu *}\left(-1+2 \delta_{L}^{d *}+2 \delta_{R}^{d *}+\delta_{L}^{u *}+\delta_{R}^{u *}\right) \Omega_{\alpha \beta}^{R *}\right\}
\end{aligned}
$$

respectively, with

$$
\Omega_{\alpha \beta}^{R}=\sum_{i, j} U_{\alpha i} \Omega_{i j}^{R} U_{\beta j}^{*}
$$


The new RH interactions do not contribute to the Dirac effective Hamiltonian in Eq. (27), which is the same as in the $\nu S M$, but they do contribute to the Majorana one in Eq. (28). Neglecting quadratic terms in the new small parameters, this reduces to

$$
\mathcal{H}_{\alpha \beta}^{M}(\lambda=-1)=\sqrt{2} G_{F} N_{e}\left\{\delta_{e \alpha} \delta_{e \beta}+\frac{N_{n}}{N_{e}} \frac{1}{2} \delta_{R}^{N \nu *} \Omega_{\alpha \beta}^{R *}\right\} .
$$

Thus, the difference between Dirac and Majorana neutrino oscillations are triggered by the extra term proportional to the neutron to electron density ratio, to $\delta_{R}^{N \nu *}$ and to the $\Omega_{\alpha \beta}^{R *}$ matrix elements. Then, to observe any difference, $\Omega_{\alpha \beta}^{R}$ can not be diagonal. It is also worth to emphasize that in contrast with other NP effects, which are quadratic in the (small) new parameters, this difference is linear in the (small) strength of the extra RH neutral interactions.

The oscillation probabilities can be found diagonalising the Hamiltonians in Eqs. (27) and (30) together with the neutrino (species) dependent kinematical term in Eq. (22) [32, 33]

$$
\mathcal{H}^{\nu}=\frac{1}{2 E_{\nu}} W\left\{\operatorname{diag}\left(\tilde{m}_{i}^{2}\right)\right\} W^{\dagger}
$$

where $W$ is the diagonalising (unitary) matrix defined by the eigenvectors of $2 E_{\nu} \mathcal{H}^{\nu}$ and $\tilde{m}_{i}^{2}$ are the corresponding (real) eigenvalues. We are now ready to calculate the transition amplitude from the initial neutrino (production) state $\mid \psi(0)>$ after travelling a distance $L$ to some other final neutrino (detection) state $|\varphi(0)\rangle$,

$$
<\varphi(0) \mid \psi(L=t)>
$$

When considering new neutrino interactions these can also affect their production and detection (see e.g. [36]). In general, to calculate the impact of NP on the full process, the modification of the initial and final states should be also taken into account. But here we are interested in the difference between Dirac and Majorana neutrino propagation, and the effects which modify in the same way both type of neutrinos can be ignored. Therefore, we can assume that neutrinos are produced and detected in the flavour states $\left|\nu_{\alpha}\right\rangle$ and $\left|\nu_{\beta}\right\rangle$, respectively. Then the transition amplitude $\nu_{\alpha} \rightarrow \nu_{\beta}$ can be written

$$
A_{\alpha \rightarrow \beta}(L)=<\nu_{\beta}(0) \mid \nu_{\alpha}(L=t)>=\left(W\left\{\operatorname{diag}\left(\exp \left[-i \frac{\tilde{m}_{i}^{2}}{2 E_{\nu}} L\right]\right)\right\} W^{\dagger}\right)_{\beta \alpha},
$$

and the transition probability [29]

$$
P_{\alpha \rightarrow \beta}(L)=\delta_{\alpha \beta}-4 \sum_{a>b} R_{\alpha \beta}^{a b} \sin ^{2} \Delta_{a b}+8 I_{\alpha \beta}^{21} \sin \Delta_{21} \sin \Delta_{31} \sin \Delta_{32},
$$


with

$$
R_{\alpha \beta}^{a b}=\operatorname{Re}\left[W_{\alpha a}^{*} W_{\beta a} W_{\alpha b} W_{\beta b}^{*}\right], \quad I_{\alpha \beta}^{21}=\operatorname{Im}\left[W_{\alpha 2}^{*} W_{\beta 2} W_{\alpha 1} W_{\beta 1}^{*}\right],
$$

and

$$
\Delta_{a b}=1.27 \frac{\left(\tilde{m}_{a}^{2}-\tilde{m}_{b}^{2}\right)\left[\mathrm{eV}^{2}\right] L[\mathrm{~km}]}{E_{\nu}[\mathrm{GeV}]}
$$

Using Eq. (26) we can obtain the transition probability for Dirac antineutrinos and Majorana neutrinos with $\lambda=+1$ from Eq. (33) with the appropriate replacements

$$
P_{\bar{\alpha} \rightarrow \bar{\beta}}(L)=P_{\alpha \rightarrow \beta}\left(L ; W \rightarrow W^{*}, G_{F} \rightarrow-G_{F}\right)
$$

\section{NUMERICAL RESULTS AND EXPERIMENTAL BOUNDS}

In this Section we quantify how large can the difference between the Dirac and Majorana neutrino oscillation probabilities be in the presence of new $\mathrm{RH}$ neutral interactions. We shall assume that $\delta_{R}^{N \nu}$ and the matrices $U^{*}$ and $\Omega^{R}$ in Eqs. (12) and (13) are real, neglecting possible CP violating phases because they do not change our results in any substantial way. For the calculations shown in the Figures below we use the first order expressions in $\delta_{R}^{N \nu} \Omega^{R}$ for the Dirac and Majorana effective Hamiltonians in Eqs. (27) and (30), respectively. We shall take $\delta_{R}^{N \nu}$ not bigger than 0.01 [37, 38], and parameterise $\Omega^{R}$ as follows

$$
\Omega_{\alpha \beta}^{R}=\left(\begin{array}{ccc}
1 & \eta & \eta^{2} \\
\eta & 1-\chi & \eta \\
\eta^{2} & \eta & 1-\omega
\end{array}\right) .
$$

We have checked that the difference of the Majorana and Dirac neutrino transition probabilities $\Delta P=P^{M}-P^{D}$ depends litlle on the diagonal elements $(\chi, \omega)$ for any choice of sign. In contrast the dependence on the off-diagonal entry $\eta$ is linear ${ }^{5}$. We choose this particular $\eta$ parameterisation of $\Omega^{R}$ only for easy comparison of the potential of the different channels to discriminate between Dirac and Majorana neutrinos. The oscillation probabilities also depend on the medium. We shall concentrate on the neutrino propagation inside Earth, allowing for variations of the travelling distance $L$ and of the neutrino energy $E_{\nu}$. The matter

\footnotetext{
${ }^{5}$ A quantitative discussion of the effects of flavour diagonal and off-diagonal non-standard Hamiltonian contributions to neutrino oscillations can be found in Ref. [26].
} 
density changes along the Earth radius, and so the electron (proton) and neutron densities in Eqs. (27) and (30). Our calculations are performed for travelling distances $L=1000,6500$ and $13000 \mathrm{~km}$ using along these paths the mean values of the matter densities, which we estimate to be equal to 3,4 and $7 \mathrm{~g} / \mathrm{cm}^{3}$, respectively. As we are only interested in illustrating the main trends, we shall not consider realistic profiles of the Earth matter density. We also need to know $U$. Using the solar, KamLAND and $\mathrm{SK}+\mathrm{K} 2 \mathrm{~K}+\mathrm{CHOOZ}$ data, the values of the five standard oscillation parameters $\theta_{12}, \theta_{13}, \theta_{23}, \delta m_{21}^{2} \equiv \delta m_{\text {sol }}^{2}$ and $\delta m_{32}^{2} \equiv \delta m_{a t m}^{2}$ can be determined. At 95\% C.L. [12]

$$
\begin{aligned}
\sin ^{2} \theta_{12} & =0.314\left(1_{-0.15}^{+0.18}\right) \\
\delta m_{21}^{2} & =7.92(1 \pm 0.09) \times 10^{-5} \mathrm{eV}^{2} \\
\sin ^{2} \theta_{23} & =0.44\left(1_{-0.22}^{+0.41}\right) \\
\delta m_{32}^{2} & =2.4\left(1_{-0.26}^{+0.21}\right) \times 10^{-3} \mathrm{eV}^{2} \\
\sin ^{2} \theta_{13} & =0.9_{-0.9}^{+2.3} \times 10^{-2}
\end{aligned}
$$

The numerical results are presented in Figs. $1-5$. We plot the values of the transition probabilities for Dirac and Majorana neutrinos. Their difference $\Delta P$ is of few per cent. Figs. 1 (a) -1 (f) prove that the transition probabilities also depend on the neutrino flavour. For $\nu_{\mu} \rightarrow \nu_{e}$ the effect first increases, and then it seems to weaken again with increasing $L$. But for the $\nu_{\mu} \rightarrow \nu_{\tau}$ channel the probability difference $\Delta P$ keeps increasing with increasing distance $L$ up to the last analyzed value $L=13000 \mathrm{~km}$. As observed in these Figures the largest effects manifest in the $\nu_{\mu} \rightarrow \nu_{\tau}$ transitions when $L=13000 \mathrm{~km}$ (approximate diameter of the Earth). In this case the largest difference $\Delta P$ between Majorana and Dirac neutrino transition probabilities increases with energy, and for $\eta=1$ reaches its maximum at $E_{\nu} \approx 57 \mathrm{GeV}$ with a long (experimentally attractive) plateau at higher energies. It varies approximately between 0.15 and 0.17 , standing for an effect on the value of $\Delta P / P^{D}$ equal to $34 \%$ at $50 \mathrm{GeV}$, increasing up to $84 \%$ at $90 \mathrm{GeV}$. It can be seen too that for small neutrino energies the transition probability difference can be significant as well. However, this is of no practical use because of the high frequency of the probability variation, which after averaging over the energy bin smooths the signal to zero. Figs. 2 (a) - (b) show the $\eta$ dependence. The effect weakens with the decrease of $\eta$ for it is proportional to $\delta_{R}^{N \nu} \eta$. Indeed, for $\eta=1 / 5$ the largest difference between the transition probabilities is reached at $E_{\nu} \approx 53 \mathrm{GeV}$, with a wide plateau too but with a similar value of $\Delta P \approx 0.035$ (see Fig. 2 

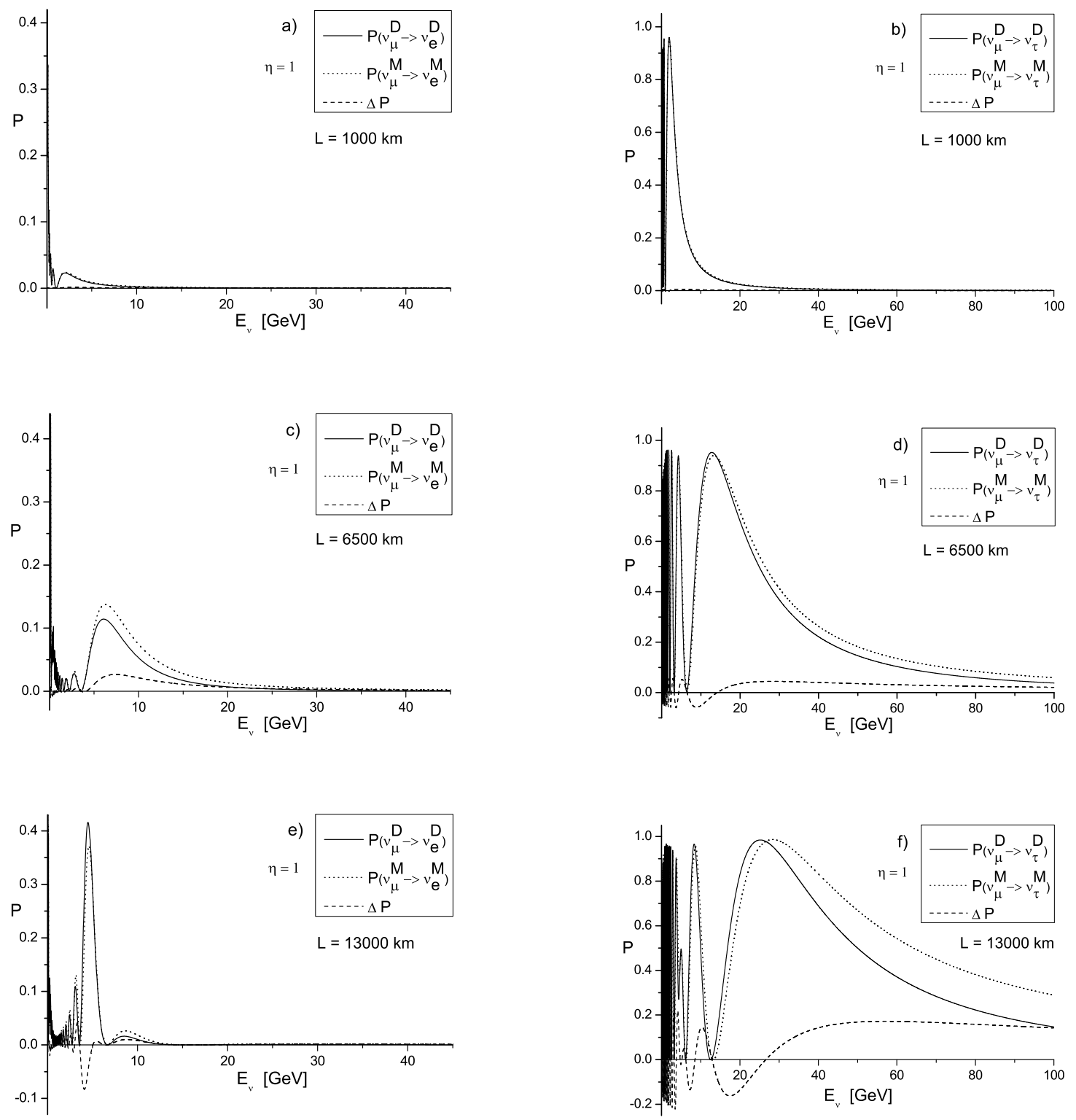

FIG. 1: Transition probabilities for Majorana and Dirac neutrinos and their difference $\Delta P$ as a function of the neutrino energy $E_{\nu} \mathrm{GeV}$ and $\eta=1$. From left to right and top to bottom: (a) $\nu_{\mu} \rightarrow \nu_{e}, L=1000 \mathrm{~km}$. (b) $\nu_{\mu} \rightarrow \nu_{\tau}, L=1000 \mathrm{~km}$. (c) $\nu_{\mu} \rightarrow \nu_{e}, L=6500 \mathrm{~km}$. (d) $\nu_{\mu} \rightarrow \nu_{\tau}$, $L=6500 \mathrm{~km}$. (e) $\nu_{\mu} \rightarrow \nu_{e}, L=13000 \mathrm{~km}$. (f) $\nu_{\mu} \rightarrow \nu_{\tau}, L=13000 \mathrm{~km}$.

(a)). To make it apparent we draw in Fig. 2 (b) the $\eta$ dependance of $\Delta P$ in the vecinity of the maximum. Thus, for $L=13000 \mathrm{~km}$ and $E_{\nu}=57 \mathrm{GeV}$ we vary $\eta$ up to 1.0. As expected the effect decreases linearly with $\eta$ vanishing for $\eta=0$. Finally, in order to visualize the 

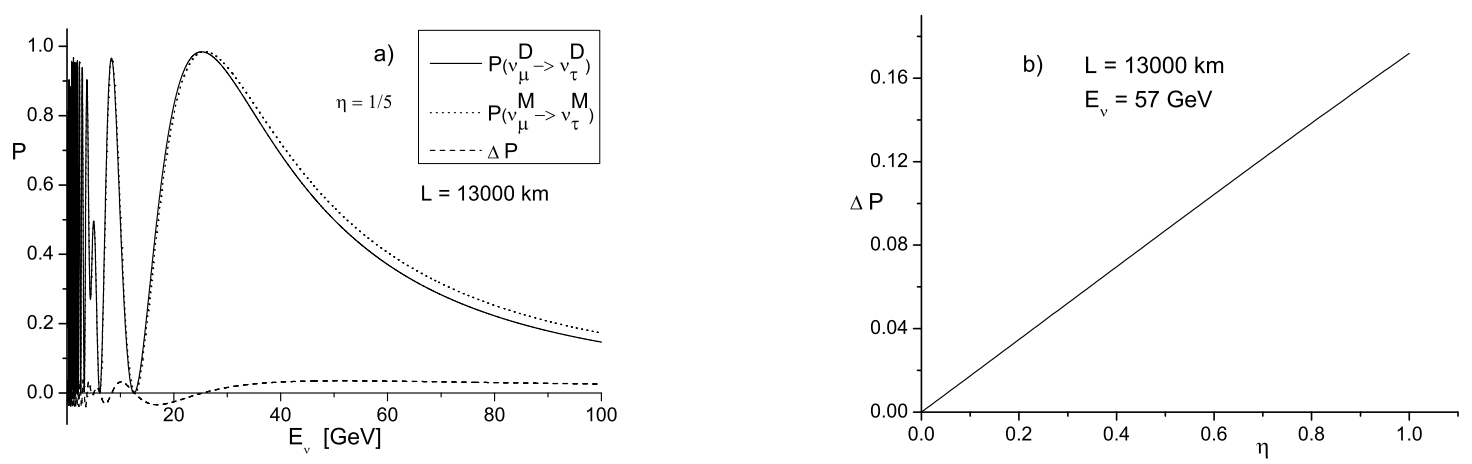

FIG. 2: Left: (a) Transition probabilities for Dirac and Majorana neutrinos and their difference $\Delta P$ as a function of the neutrino energy $E_{\nu}$ in $\mathrm{GeV}$ for $L=13000 \mathrm{~km}$ and for the process $\nu_{\mu} \rightarrow \nu_{\tau}$, with $\eta=1 / 5$. Right: (b) Transition probability difference $\Delta P$ for the process $\nu_{\mu} \rightarrow \nu_{\tau}$ as a function of $\eta$ for a neutrino energy $E_{\nu}=57 \mathrm{GeV}$ and $L=13000 \mathrm{~km}$.

two-dimensional dependance of $\Delta P$ on $L$ and $E_{\nu}$ we include Fig. 3. For $E_{\nu} \leq 20 \mathrm{GeV}, \Delta P$ changes very rapidly as a function of the baseline $L$. For larger energies the variation is slower.

The question arises whether current experimental errors [10, 11, 12] are not too weak to prevent the transition probabilities for Dirac and Majorana neutrinos from overlapping. We show in Fig. 4 (a) the $\nu_{\mu} \rightarrow \nu_{\tau}$ transition probabilities for present values of the oscillation parameters (Eq. (38)). The bands are obtained varying the oscillation parameters within their $95 \%$ C.L. limits. Indeed, they are too wide to be able to distinguish between Dirac and Majorana neutrinos. However, future experiments will provide more precise measurements. Hence, we plot in Fig. 4 (b) the bands for the same central values of the oscillation parameters but assuming that the errors are reduced by a factor of 5 . In this case the Majorana (upper) and Dirac (lower) bands separate, allowing in principle to distinguish between both types of neutrinos. Obviously varying $\eta$ (and the form of $\Omega$ ) one can make the effect much smaller and unobservable.

This encourages to search for the neutrino character at future experiments. One must be aware, however, that not only the experimental errors of the standard oscillation parameters must be significantly reduced but they must be determined independently to avoid new confusion [39]. A relevant related comment is that the observation of deviations from the SM 


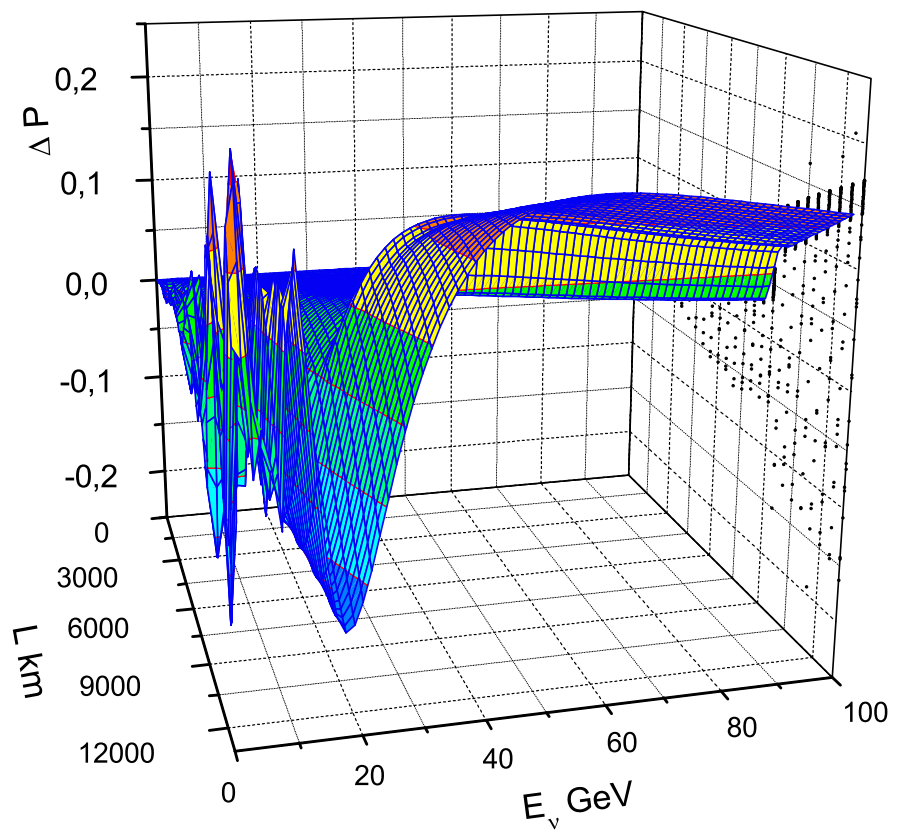

FIG. 3: Transition probability difference $\Delta P$ as a function of the neutrino energy $E_{\nu}$ in $\mathrm{GeV}$ and the distance $L$ in $\mathrm{km}$ for the process $\nu_{\mu} \rightarrow \nu_{\tau}$ with $\eta=1$. The calculations are performed for matter densities equal to 3,4 and $7\left[\mathrm{~g} / \mathrm{cm}^{3}\right]$ and travelling distances $L$ between $0-4500,5000-9000$ and $9500-13000 \mathrm{~km}$, respectively. The projections on the $L-\Delta P$ plane are drawn with dots.

predictions, as those drawn for Majorana neutrinos in the presence of $\mathrm{RH}$ neutral currents in Fig. 4, can have their origin in the NP previously discussed or be a manifestation of the inverted character of the neutrino mass hierarchy. The distances and energies relevant in our case are similar to those sensitive to atmospheric neutrino oscillations, and then to $\delta m_{32}^{2}$. Its sign, which is unknown at present and may be positive (normal) or negative (inverted scheme), can not be fixed in the $\nu_{\mu} \rightarrow \nu_{\tau}$ channel within the $\nu S M^{6}$. Therefore in Fig. 5 we plot the same transition probabilities as in Fig. 4 (b) but for the inverted scheme. The central lines and future bands for Dirac neutrinos, which give the same predictions as the $\nu S M$, almost coincide for both sign assignments. In contrast, the band for Majorana

\footnotetext{
$\overline{{ }^{6} \text { If } \theta_{13}}$ is sizeable, the sign of $\delta m_{a t m}^{2}$ will be possibly established in $\nu_{\mu} \rightarrow \nu_{e}$ oscillation experiments [40], even if there are no new interactions. In our case the relevant channel is $\nu_{\mu} \rightarrow \nu_{\tau}$ because it is where the effect of new RH neutral interactions is larger.
} 

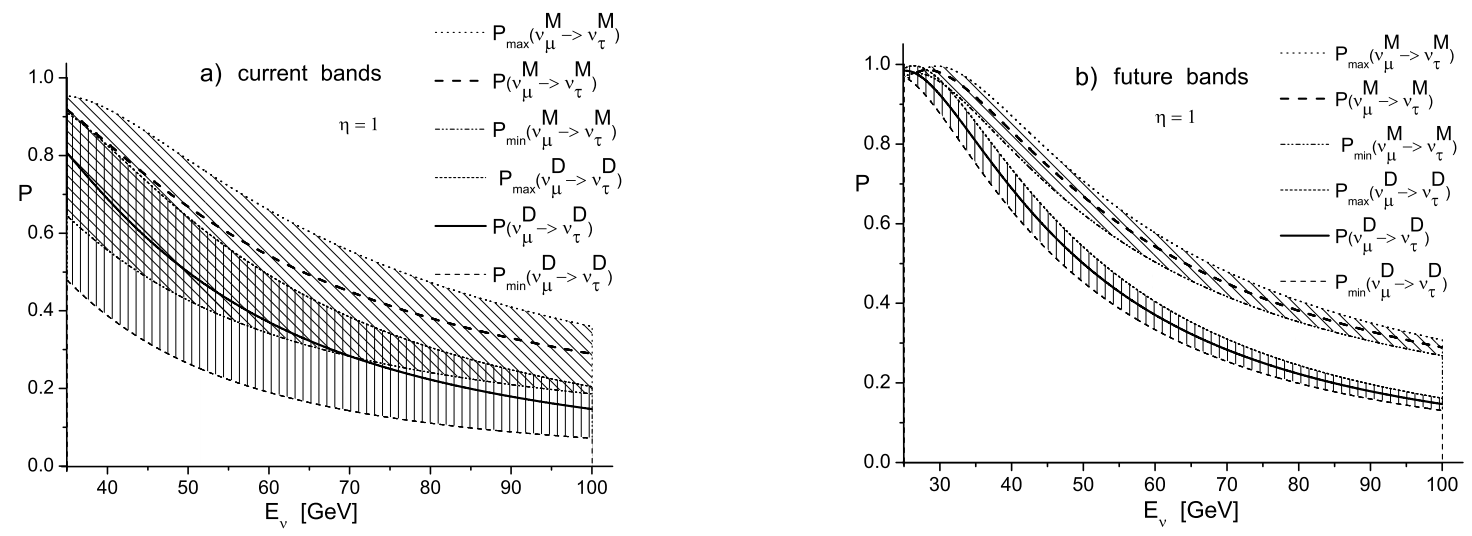

FIG. 4: Upper and lower bands corresponding to transition probabilities for Dirac and Majorana neutrino, respectively, as a function of the neutrino energy $E_{\nu}$ in $\mathrm{GeV}$ for $L=13000 \mathrm{~km}$ and the process $\nu_{\mu} \rightarrow \nu_{\tau}$ with $\eta=1$. Left: (a) For current $95 \%$ confidence intervals of the oscillation parameters [12]. Right: (b) For future $95 \%$ confidence intervals of the oscillation parameters (assuming a statistics $\approx 25$ times larger than nowadays).

neutrinos moves below (above) the Dirac neutrino band for the inverted (normal) hierarchy, reversing the sign of $\Delta P$ but maintaining its size to a large extent. The same displacement is obtained changing the sign of $\eta$. This is well-known [41]. The sign of $\delta m_{a t m}^{2}$ can be fixed if there is other contribution to the interaction potential energy with a well-defined sign, with which the standard contribution can interfere. In our case the extra piece is provided by the new $\mathrm{RH}$ interactions. If in the future any of the two schemes is established, the observation of the Majorana character of the light neutrinos in the process in Figs. 4 (b) and 5 would also allow for the determination of the sign of $\eta$.

\section{CONCLUSIONS AND MODELS}

Within the $\nu S M$ oscillations of relativistic neutrinos do not differentiate between Dirac and Majorana neutrinos. This is not in general the case beyond the $\nu S M$, and Dirac and Majorana neutrinos can be differentiated by how they propagate in matter. Indeed, extended models with new RH currents or tensor terms do distinguish between both types of neutrinos. However, the spin-flip tensor transitions require a polarised medium to manifest 


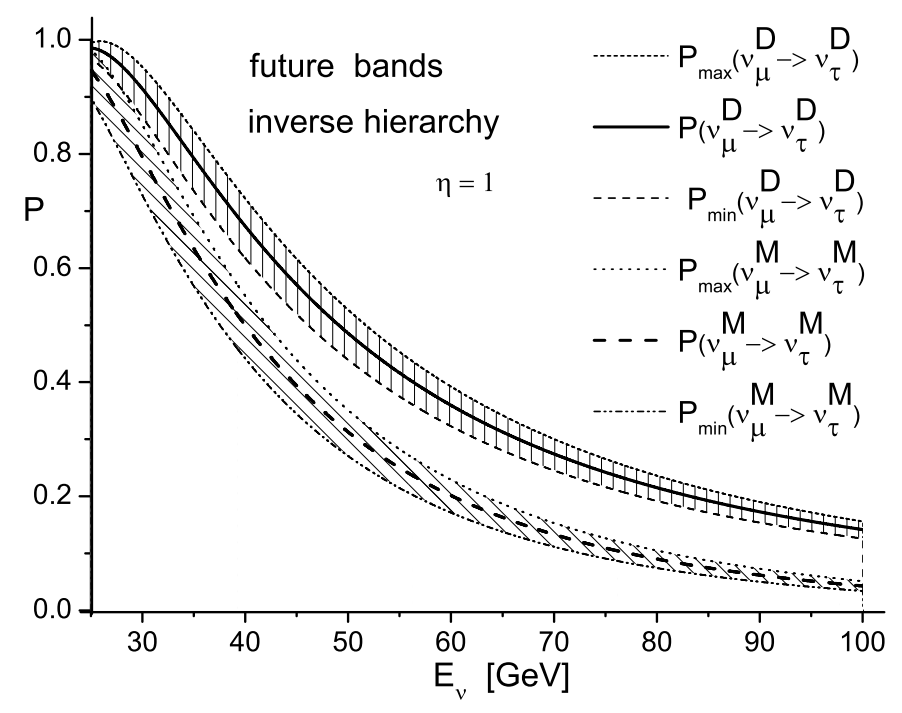

FIG. 5: Transition probabilities for Dirac (upper band) and Majorana (lower band) neutrinos in the inverse mass scheme (assuming a statistics $\approx 25$ times larger than nowadays). There is almost no difference between the Dirac neutrino bands for the inverted and normal schemes (see Fig. 4 (b)).

[27]. Moreover, the production and detection of (anti)neutrinos of the wrong helicity are strongly suppressed. Hence, we concentrated on the analysis of additional RH currents which allow for unsuppressed spin non-flip transitions. This NP does not modify the oscillation of Dirac neutrinos in matter for we can assign to them a definite lepton number, but it does modify the propagation of Majorana neutrinos for they participate of both types (LH and $\mathrm{RH}$ ) of currents. The largest effect, which is linear in the new small parameters, is associated to extra RH neutral currents. However, it is needed a non-trivial flavour structure to differentiate Dirac from Majorana neutrino oscillations in matter. Both LH and RH neutral current interactions can not be simultaneously diagonal.

Numerical estimates show that the largest differences between Majorana and Dirac neutrino oscillations manifest in the $\nu_{\mu} \rightarrow \nu_{\tau}$ channel. This difference between both probabilities can be as large as $\sim 0.16$ for the normal as well as for the inverted scheme, but with opposite sign. Unfortunately, this large effect holds for the channel which is more difficult to measure and for a very large baseline $L \simeq 13000 \mathrm{~km}$. Smaller effects are visible for $\nu_{\mu} \rightarrow \nu_{e}$ and other 
baselines.

The large effect found is a consequence of the linear, and then unsuppressed, dependence of the transition probabilities for Majorana neutrinos on the new RH neutral currents. This, which is our main observation, relies on two ingredients for being in practice of some relevance. The strength of the new flavour violating RH transitions has to be sufficiently large, and the precision reached in oscillation experiments sufficiently high. We have not attempted a global fit to determine the present experimental limits on the new (flavour changing) couplings but we have looked for a class of $\nu S M$ extensions which may accommodate such new terms. Let us present a simple example of a class of extended gauge models of possible cosmological interest [42]. Consider an extended gauge group with an extra $U(1)_{N}$ under which all known particles transform trivially, and enlarge the matter content with two new fermion singlets under the $\nu S M$ but with $U(1)_{N}$ charges 2 and -2 (what makes the model free of gauge anomalies) and the same lepton number (what forbids direct mass terms in the Dirac case). This is completed with two extra Higgs doublets $h_{i}^{\prime}$ and an extra Higgs singlet $\phi$ with $U(1)_{N}$ charges $2,-2$ and 1, respectively. The new fermions get their masses through their very small Yukawa couplings with the $\nu S M$ neutrinos, once $h_{i}^{\prime}$ get non-zero vacuum expectation values (vev) $v_{i}^{\prime}$, also (in principle) much smaller than the $\nu S M$ Higgs vev $\langle h\rangle=v$. Whereas the singlet vev $\langle\phi\rangle=x$ is (much) larger than $v$, and gives a mass to the new gauge boson. The new RH neutral current term for the light neutrinos is proportional to the $Z$ boson mixing with the new gauge boson, which also scales with $v_{i}^{\prime}$. The $\nu S M$ is practically recovered for $\left\langle h_{i}^{\prime}\right\rangle=0$. Then, the new phenomenology of this model depends on those vev. In the absence of Majorana masses lepton number is conserved and there are one massless $(\mathrm{LH})$ neutrino and two light Dirac neutrinos. If there are also light neutrino masses, we have five light Majorana neutrinos. In the first case the sum in the extra $\mathrm{RH}$ piece is over the new $\mathrm{RH}$ fermions only, while in the second one the sums are over all the Majorana neutrinos ${ }^{7}$. One can write down more complicated models with at least three massive Dirac neutrinos. Nevertheless, the important question is if the new gauge boson and the extra scalars can escape detection and at the same time the LH interactions approach the $\nu S M$ ones, as required by experiment, whereas the new $\mathrm{RH}$ piece has flavour changing couplings large enough, $\sim 100$ times smaller than the standard couplings. The

\footnotetext{
7 The LH charged current sum also extends to the new light neutrinos.
} 
main tension manifests in the Majorana case because the effective RH couplings are the product of the $Z$ mixing with the new gauge boson times the square of the mixing of the standard neutrinos with the new singlets, and none of them can be negligible. However, the usual limits can not be directly applied for the new gauge boson couples weakly (through its mixing with the standard $Z$ boson) to charged fermions and the induced flavour violation is proportional to the tiny neutrino masses ${ }^{8}$. Obviously, a detailed analysis of the present experimental constraints from precise electroweak data [43] is necessary to decide on the allowed region of parameters. Such a study is beyond the scope of this paper.

Our generic conclusion is that we should be aware of the possibility that NP may allow to differentiate between Dirac and Majorana neutrinos propagating in matter, and that it is worth to search for this difference in the next generation of very precise neutrino oscillation experiments. A positive signal would not only indicate the Majorana character of massive neutrinos but the existance of new interactions.

Ackowledgments: We thank A. Bueno and J. Santiago for a careful reading of the manuscript and useful comments. This work has been partially supported by Polish Ministry of Science under Grant 1 P03 B 049 26, by MEC (FPA2006-05294), by Junta de Andalucía (FQM 101 and FQM 437), and by the European Community's Marie-Curie Research Training Network under contract MRTN-CT-2006-035505 "Tools and Precision Calculations for Physics Discoveries at Colliders".

[1] B. T. Cleveland et al., Astrophys. J. 496, 505 (1998); Y. Fukuda et al. [Kamiokande Collaboration], Phys. Rev. Lett. 77, 1683 (1996); V. N. Gavrin [SAGE Collaboration], Nucl. Phys. Proc. Suppl. 91, 36 (2001); W. Hampel et al. [GALLEX Collaboration], Phys. Lett. B 447, 127 (1999); M. Altmann et al. [GNO Collaboration], Phys. Lett. B 490, 16 (2000) arXiv:hep-ex/0006034]; S. Fukuda et al. [Super-Kamiokande Collaboration], Phys. Rev. Lett.

\footnotetext{
${ }^{8}$ Such an effective RH neutral coupling requires a relatively large neutrino mixing with the new light fermions, as well as a relatively large gauge boson mixing. Even for a slight deviation of $\varepsilon_{L}^{C} U_{\alpha i}^{*}$ in Eq. (12) from unitarity, $\varepsilon_{L}^{C}=0.98$, the former can be a priori as large as 0.2 . However, the latter is strongly constrained by the bound on the $\rho$ parameter. At any rate, the experimental limits on this $Z^{\prime}$ are weaker than the typical ones on extra gauge bosons [43], as they are its couplings to standard fermions [44].
} 
86, 5651 (2001) arXiv:hep-ex/0103032; Q. R. Ahmad et al. [SNO Collaboration], Phys. Rev. Lett. 87, 071301 (2001) arXiv:nucl-ex/0106015]; Q. R. Ahmad et al. [SNO Collaboration], Phys. Rev. Lett. 89, 011301 (2002) arXiv:nucl-ex/0204008]; S. N. Ahmed et al. [SNO Collaboration], Phys. Rev. Lett. 92, 181301 (2004) arXiv:nucl-ex/0309004; K. Eguchi et al. [KamLAND Collaboration], Phys. Rev. Lett. 90, 021802 (2003) arXiv:hep-ex/0212021]; Y. Fukuda et al. [Super-Kamiokande Collaboration], Phys. Rev. Lett. 81, 1562 (1998) arXiv:hep-ex/9807003; W. W. M. Allison et al., Phys. Lett. B 391, 491 (1997) arXiv:hep-ex/9611007]; W. W. M. Allison et al. [Soudan-2 Collaboration], Phys. Lett. B 449, 137 (1999) arXiv:hep-ex/9901024; for reviews on solar neutrinos J. Bahcall's URL

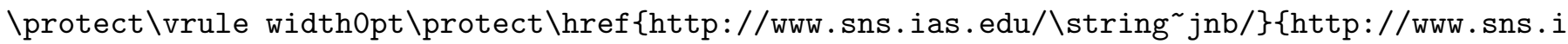
on atmospheric neutrinos C. K. Jung, C. McGrew, T. Kajita and T. Mann, Ann. Rev. Nucl. Part. Sci. 51, 451 (2001); see also $\backslash$ protect \vrule width0pt\protect \href\{http://neutrinooscillation.org/\}\{http://neutrinooscil

[2] E. Majorana, Nuovo Cim. 14, 171 (1937).

[3] S. M. Bilenky and S. T. Petcov, Rev. Mod. Phys. 59, 671 (1987) [Erratum-ibid. 61, 169 (1989)]; B. Kayser, F. Gibrat-Debu and F. Perrier, The Physics of massive neutrinos, World Sci. Lect. Notes Phys. 25, 1 (1989).

[4] R. N. Mohapatra and P. B. Pal, Massive neutrinos in physics and astrophysics. Second edition, World Sci. Lect. Notes Phys. 60, 1 (1998) [World Sci. Lect. Notes Phys. 72, 1 (2004)].

[5] S.M. Bilenky, A. Faessler, F. Simkovic, S. M. Bilenky, A. Faessler and F. Simkovic, Phys. Rev. D 70, 033003 (2004) arXiv:hep-ph/0402250]; S. M. Bilenky, Phys. Atom. Nucl. 69, 2134 (2006) arXiv:hep-ph/0509098; S. T. Petcov, Phys. Scripta T121, 94 (2005) arXiv:hep-ph/0504166]; A. S. Barabash, JINST 1, P07002 (2006) arXiv:hep-ex/0602037; M. Hirsch, A. Villanova del Moral, J. W. F. Valle and E. Ma, Phys. Rev. D 72, 091301 (2005) [Erratum-ibid. D 72, 119904 (2005)] arXiv:hep-ph/0507148]; J. W. F. Valle, Nucl. Phys. Proc. Suppl. 145, 141 (2005) arXiv:hep-ph/0508067].

[6] E.M. Lyman, Phys. Rev. 55, 234(A) (1939); R.O. Haxby, W.E. Shoupp, W.E. Stephens and W.H. Wells, Phys. Rev. 58, 1035 (1940).

[7] R. Abela, M. Daum, G. H. Eaton, R. Frosch, B. Jost, P. R. Kettle and E. Steiner, Phys. Lett. B 146, 431 (1984); H. B. Anderhub et al., Phys. Lett. B 114, 76 (1982).

[8] F. Maltoni, J. M. Niczyporuk and S. Willenbrock, Phys. Rev. Lett. 86, 212 (2001) 
arXiv:hep-ph/0006358.

[9] M. Czakon, J. Gluza and M. Zralek, Phys. Lett. B 465, 211 (1999) arXiv:hep-ph/9906381; S. Pascoli and S. T. Petcov, Phys. Lett. B 544, 239 (2002) arXiv:hep-ph/0205022; S. M. Bilenky, A. Faessler, T. Gutsche and F. Simkovic, Phys. Rev. D 72, 053015 (2005) [arXiv:hep-ph/0507260]; M. Czakon, J. Gluza, J. Studnik and M. Zralek, Phys. Rev. D 65, 053008 (2002) arXiv:hep-ph/0110166; A. Merle and W. Rodejohann, Phys. Rev. D 73, 073012 (2006) arXiv:hep-ph/0603111; B. Dziewit, K. Kajda, J. Gluza and M. Zralek, Phys. Rev. D 74, 033003 (2006) arXiv:hep-ph/0604193.

[10] M. C. Gonzalez-Garcia and M. Maltoni, arXiv:hep-ph/0406056; M. C. Gonzalez-Garcia, Phys. Scripta T121, 72 (2005) arXiv:hep-ph/0410030].

[11] B. Aharmim et al. [SNO Collaboration], Phys. Rev. C 72, 055502 (2005) arXiv:nucl-ex/0502021.

[12] G. L. Fogli, E. Lisi, A. Marrone and A. Palazzo, Prog. Part. Nucl. Phys. 57, 742 (2006) arXiv:hep-ph/0506083.

[13] B. Pontecorvo, Sov. Phys. JETP 6, 429 (1957) [Zh. Eksp. Teor. Fiz. 33, 549 (1957)].

[14] M. Zralek, Acta Phys. Polon. B 28, 2225 (1997) arXiv:hep-ph/9711506; M. Zralek, Acta Phys. Polon. B 29, 3925 (1998) arXiv:hep-ph/9810543]; M. Czakon, M. Zralek and J. Gluza, Acta Phys. Polon. B 30, 3121 (1999) arXiv:hep-ph/9910357.

[15] S. M. Bilenky, J. Hosek and S. T. Petcov, Phys. Lett. B 94, 495 (1980).

[16] M. Doi, T. Kotani, H. Nishiura, K. Okuda and E. Takasugi, Phys. Lett. B 102, 323 (1981).

[17] L. Wolfenstein, Phys. Rev. D 17, 2369 (1978); S. P. Mikheev and A. Y. Smirnov, Nuovo Cim. C 9, 17 (1986).

[18] S. P. Rosen and J. M. Gelb, Phys. Rev. D 34, 969 (1986); C. W. Kim, S. Nussinov and W. K. Sze, Phys. Lett. B 184, 403 (1987); A. Baldini and G. F. Giudice, Phys. Lett. B 186, 211 (1987); T. K. Kuo and J. T. Pantaleone, Phys. Lett. B 198, 406 (1987); D. Notzold and G. Raffelt, Nucl. Phys. B 307, 924 (1988).

[19] F. del Aguila and M. Zralek, Acta Phys. Polon. B 33, 2585 (2002) arXiv:hep-ph/0207319.

[20] K. Kiers and N. Weiss, Phys. Rev. D 56, 5776 (1997) arXiv:hep-ph/9704346.

[21] F. Boehm and P. Vogel, Physics of massive neutrinos, Cambridge University Press, (1992).

[22] J. W. F. Valle, Phys. Lett. B 199, 432 (1987); J. W. F. Valle, J. Phys. G 29, 1819 (2003); and references therein. 
[23] M. C. Gonzalez-Garcia et al., Phys. Rev. Lett. 82, 3202 (1999) arXiv:hep-ph/9809531.

[24] N. Fornengo, M. Maltoni, R. T. Bayo and J. W. F. Valle, Phys. Rev. D 65, 013010 (2002) arXiv:hep-ph/0108043.

[25] M. Garbutt and B. H. J. McKellar, arXiv:hep-ph/0308111.

[26] M. Blennow, T. Ohlsson and W. Winter, arXiv:hep-ph/0508175.

[27] S. Bergmann, Y. Grossman and E. Nardi, Phys. Rev. D 60, 093008 (1999) arXiv:hep-ph/9903517.

[28] W. Buchmuller and D. Wyler, Nucl. Phys. B 268, 621 (1986); C. Arzt, M. B. Einhorn and J. Wudka, Nucl. Phys. B 433, 41 (1995) arXiv:hep-ph/9405214].

[29] J. Gluza and M. Zralek, Phys. Lett. B 517, 158 (2001) arXiv:hep-ph/0106283.

[30] C. Giunti and C. W. Kim, Found. Phys. Lett. 14, 213 (2001) arXiv:hep-ph/0011074.

[31] Z. Maki, M. Nakagawa and S. Sakata, Prog. Theor. Phys. 28, 870 (1962).

[32] C. W. Kim and A. Pevsner, Neutrinos in physics and astrophysics, Harwood Academic Publishers, Contemp. Concepts Phys. 8, 1 (1993).

[33] B. Bekman, J. Gluza, J. Holeczek, J. Syska and M. Zralek, Phys. Rev. D 66, 093004 (2002) arXiv:hep-ph/0207015.

[34] F. del Aguila, J. A. Aguilar-Saavedra and R. Pittau, J. Phys. Conf. Ser. 53, 506 (2006) arXiv:hep-ph/0606198.

[35] P. Langacker, J. P. Leveille and J. Sheiman, Phys. Rev. D 27, 1228 (1983); S. P. Mikheev and A. Y. Smirnov, Sov. J. Nucl. Phys. 42, 913 (1985) [Yad. Fiz. 42, 1441 (1985)]; T. K. Kuo and J. T. Pantaleone, Rev. Mod. Phys. 61, 937 (1989); J. F. Nieves, Phys. Rev. D 40, 866 (1989); P. B. Pal and T. N. Pham, Phys. Rev. D 40, 259 (1989); W. Grimus and T. Scharnagl, Mod. Phys. Lett. A 8, 1943 (1993); J. C. D’Olivo and J. F. Nieves, Phys. Lett. B 383, 87 (1996) arXiv:hep-ph/9512428; H. Nunokawa, V. B. Semikoz, A. Y. Smirnov and J. W. F. Valle, Nucl. Phys. B 501, 17 (1997) arXiv:hep-ph/9701420.

[36] M. C. Gonzalez-Garcia, Y. Grossman, A. Gusso and Y. Nir, Phys. Rev. D 64, 096006 (2001) arXiv:hep-ph/0105159.

[37] W. Sobkow, Acta Phys. Polon. B 31, 1709 (2000) arXiv:hep-ph/0002289].

[38] M. C. Gonzalez-Garcia and M. Maltoni, Phys. Rev. D 70, 033010 (2004) arXiv:hep-ph/0404085.

[39] P. Huber, T. Schwetz and J. W. F. Valle, Phys. Rev. D 66, 013006 (2002) 
arXiv:hep-ph/0202048.

[40] D. S. Ayres et al. [NOvA Collaboration], arXiv:hep-ex/0503053.

[41] B. Kayser, In the Proceedings of 32nd SLAC Summer Institute on Particle Physics (SSI 2004): Natures Greatest Puzzles, Menlo Park, California, 2-13 Aug 2004, pp L004 arXiv:hep-ph/0506165.

[42] P. H. Gu and H. J. He, JCAP 0612, 010 (2006) arXiv:hep-ph/0610275; K. Cheung and T. C. Yuan, arXiv:hep-ph/0701107; D. Hooper, arXiv:hep-ph/0701194.

[43] W. M. Yao et al. [Particle Data Group], J. Phys. G 33, 1 (2006).

[44] F. del Aguila, Acta Phys. Polon. B 25, 1317 (1994) arXiv:hep-ph/9404323]; F. del Aguila, M. Cvetic and P. Langacker, Phys. Rev. D 52, 37 (1995) arXiv:hep-ph/9501390. 